Copyright by the American Physical Society. Feng, G. F.; Zallen, R.; Epp, J. M.; Dillard, J. G., "Raman-scattering and optical studies of argon-etched GaAs surfaces," Phys. Rev. B 43, 9678 DOI: http://dx.doi.org/10.1103/PhysRevB.43.9678

\title{
Raman-scattering and optical studies of argon-etched GaAs surfaces
}

\author{
G. F. Feng* and R. Zallen \\ Department of Physics, Virginia Tech, Blacksburg, Virginia 24061 \\ J. M. Epp ${ }^{\dagger}$ and J. G. Dillard \\ Department of Chemistry, Virginia Tech, Blacksburg, Virginia 24061
}

(Received 26 November 1990)

\begin{abstract}
We have studied the structual damage in low-energy argon-ion-bombarded (ion-etched) GaAs using Raman scattering and ultraviolet reflectivity. When combined with post-bombardment sequential chemical etching, the Raman results reveal a graded depth profile of the damage layer, with a nearly linear damage dropoff with depth. The total damage-layer thickness is about $600 \AA$ for high-fluence bombardment with $3.89-\mathrm{keV} \mathrm{Ar}^{+}$ions. The spectral effects produced by argon etching are very different from those produced by high-energy ion implantation. The longitudinal-optic Raman line seen for argon-etched GaAs is not shifted and broadened as in ion-implanted GaAs. More striking are the results of the reflectivity measurements. For argon-etched GaAs, the electronic interband peaks are both broadened and strongly red shifted relative to the crystal peaks; for ionimplanted GaAs, only the broadening occurs. Distinct nanocrystals, which account for the effects seen in ion-implanted GaAs, are evidently absent in argon-etched GaAs. Instead, the damage layer caused by argon etching appears to be characterized by a very high density of point defects, which previous work suggests may be arsenic vacancies.
\end{abstract}

\section{INTRODUCTION}

Ion etching is a low-energy ion-bombardment process in which substrate surface atoms are removed (sputtered) via momentum transfer from incident energetic particles. Structural damage occurs during ion etching due to collisions between the incident ions and the near-surface atoms, and subsequent cascades. In this paper we describe a study of the distribution and the nature of the near-surface structural damage in argon-etched gallium arsenide $\left(\mathrm{Ar}^{+}\right.$-etched GaAs).

Ion-etching-induced damage in semiconductors has been studied by techniques such as electrical $(I-V, C-V)$ characterization, ${ }^{1,2}$ secondary-ion mass spectrometry, ${ }^{3,4}$ spectroscopic ellipsometry, ${ }^{3}$ and Raman spectroscopy. ${ }^{5}$ The present paper presents the results of a combination of experiments involving argon-ion bombardment, Raman-scattering measurements, ultraviolet (uv) reflectivity measurements, and wet-chemical etching for depth profiling. Our Raman measurements on chemically etched, $\mathrm{Ar}^{+}$-etched $\mathrm{GaAs}$ show the structural damage to be concentrated in a thin (a few hundred angstroms) near-surface layer. We were able to quantitatively follow the recovery of the intensity of the longitudinal-optic (LO) Raman line at $292 \mathrm{~cm}^{-1}$, with the sequential removal of damage-layer material by the wet-chemical etch. When the underlying undamaged substrate is reached, the LO-line intensity is restored to the level characteristic of crystalline GaAs (c-GaAs). A graded damage profile, derived from a model which includes the damage distribution produced by individual ions and the sputtering away of surface material, is found to fit the observed dependence of the LO intensity on the chemical-etch depth. Unlike the case of high-energy ion implantation, the LO line observed for the low-energy argonbombarded material does not shift or broaden appreciably. However, in the uv reflectivity studies (uv denotes generically, in this paper, the visible and near-ultraviolet regime from 1.6 to $5.6 \mathrm{eV}$ ) of electronic interband transitions, we observe substantial red shifts for the interband peak positions in $\mathrm{Ar}^{+}$-etched GaAs relative to the peak positions in $c$-GaAs. This, too, is in marked contrast to the situation for ion-implanted GaAs. Low-energy etching and high-energy implantation evidently produce damage layers that are structurally quite different.

Our experimental procedures are described in Sec. II, and Raman-scattering-based results obtained for the depth profile of the damage layer are presented in Sec. III. Implications for the structural nature of the damage, based on both the uv reflectivity and the Raman data, are discussed in Sec. IV. Our main findings are summarized in Sec. V.

\section{EXPERIMENT}

The virgin gallium arsenide samples were silicondoped, (liquid-encapsulated-Czochralski-) (LEC-) grown, (100)-oriented, single-crystal wafers. The wafers were obtained from the Morgan Semiconductor Division of Ethyl Corporation. The samples were $n$ type with carrier concentration in the range $(2-7) \times 10^{16} \mathrm{~cm}^{-3}$. The surfaces were of good optical quality, and their uv reflectivity exhibited the sharp spectral features characteristic of crystalline GaAs. Prior to ion-beam exposure, the wafers were cleaned in a $1: 1 \mathrm{HCl}$ solution to remove surface contaminants, and then rinsed in deionized water. 
Argon-ion bombardment was carried out in a Perkin Elmer PHI 5300 x-ray photoelectron spectrometer (XPS) system. The incident ion energy was varied between 0.5 and $3.89 \mathrm{keV}$; the ion fluence was fixed at $1 \times 10^{17}$ ions $/ \mathrm{cm}^{2}$ for all ion-etched samples in this study. The angle of incidence of the ion beam was about $45^{\circ}$ from the surface normal, in a nonchanneling direction not far from a $\langle 111\rangle$ axis. The bombardment parameters are listed in Table I. Bombardment was performed at room temperature. The beam was raster scanned over an area of $10 \times 10 \mathrm{~mm}^{2}$. The base pressure in the chamber was about $2 \times 10^{-8}$ Torr, and it rose to $10^{-7}$ Torr during ion bombardment. Because of the low ion currents used in these experiments (see Table I), the temperature rise during ion bombardment was negligible. After ion bombardment, the samples were exposed to air during the spectroscopic measurements.

In order to depth profile the surface damage, the ionbombarded samples were chemically etched using a weak acidic solution 1:1:100 $\quad \mathrm{H}_{2} \mathrm{SO}_{4}: 30 \% \mathrm{H}_{2} \mathrm{O}_{2}: \mathrm{H}_{2} \mathrm{O} .{ }^{6,7}$ The etch rate had been studied previously, yielding a rate of about $530 \AA / \mathrm{min}$ for $c$-GaAs and slightly higher for ionimplantation-damaged GaAs. ${ }^{7}$ The etch rate was assumed to be $100 \AA /(10 \mathrm{sec})$ in this study for both the near-surface high-damage region and the less-damaged region further away from the surface. The shortest etch time was $10 \mathrm{sec}$, corresponding to the removal of a layer of about $100 \AA$. Due to the approximate etch rate and the short etch times, there is a relatively large etch-depth uncertainty; the error is estimated to be about $20 \%$ of the etched depth.

Room-temperature Raman-scattering measurements were carried out using a double-monochromator spectrometer (SPEX 1403) and with argon-ion and kryptonion lasers. A near-backscattering geometry was used. ${ }^{7}$ The incident beam was polarized perpendicular to the incident plane; the scattered light of both polarizations was collected. Zinc-blende-structure selection rules cause the transverse-optic (TO) Raman line to be suppressed in this geometry, ${ }^{8}$ so that the LO line dominates the spectrum. Normalized Raman-intensity measurements were made using a replacement technique ${ }^{7}$ with either $c$-GaAs or $\mathrm{CaF}_{2}$ as reference standard. Stokes spectra are presented in this paper.

Optical reflectivity spectra in the visible and nearultraviolet region (abbreviated here generically as uv) were taken at room temperature. The dielectric function and other optical functions were derived from the observed reflectivity spectrum using Lorentz-oscillator analysis and curve-fitting techniques described previous1y. ${ }^{9}$

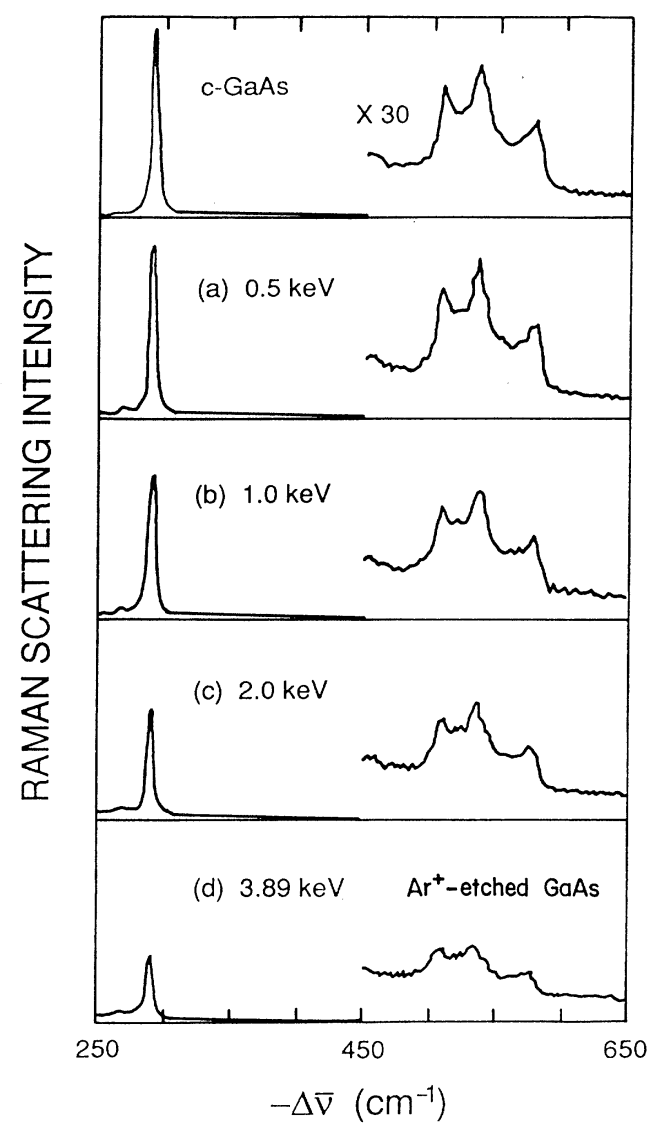

FIG. 1. Raman scattering spectra of the $\mathrm{Ar}^{+}$-bombarded GaAs samples. The top panel shows the spectrum of crystalline GaAs ( $c$-GaAs) as a reference. The kinetic energy of the bombarding ions is given with each spectrum. Raman-scattering intensity is plotted against the wave-number downshift $(-\Delta v)$ relative to the laser line.

\section{DAMAGE PROFILE}

Raman spectra of the series of $\mathrm{Ar}^{+}$-bombarded GaAs samples are shown in Fig. 1. Raman intensities are normalized to the same reference standard, so that changes in peak heights are significant. The spectra were taken with the $4579-\AA$ (2.71-eV) line of the argon-ion laser. The laser-beam probing depth (optical penetration depth $\left.d_{\text {opt }}\right)$ at this photon energy, $1 /(2 \alpha)$, where $\alpha$ is the absorption coefficient, is about $250 \AA$ for $c$-GaAs, ${ }^{10}$ about $100 \AA$ for $a$-GaAs, ${ }^{11}$ and in between for the damaged samples. ${ }^{9}$

TABLE I. Experimental parameters for the argon-etched samples.

\begin{tabular}{ccccc}
\hline Sample & $\begin{array}{c}\text { Fluence } \\
\left(\text { ions } / \mathrm{cm}^{2}\right)\end{array}$ & $\begin{array}{c}\text { Energy } \\
(\mathrm{keV})\end{array}$ & $\begin{array}{c}\text { Current } \\
(\mu \mathrm{A})\end{array}$ & $\begin{array}{c}\text { Bombardment } \\
\text { time }\end{array}$ \\
\hline$(\mathrm{a})$ & $1 \times 10^{17}$ & 0.5 & 0.28 & $15 \mathrm{~h} 52 \mathrm{~min}$ \\
$(\mathrm{~b})$ & $1 \times 10^{17}$ & 1.0 & 1.4 & $3 \mathrm{~h} 10 \mathrm{~min}$ \\
$(\mathrm{c})$ & $1 \times 10^{17}$ & 2.0 & 16 & $16 \mathrm{~min} 40 \mathrm{sec}$ \\
$(\mathrm{d})$ & $1 \times 10^{17}$ & 3.89 & 36 & $7 \mathrm{~min} 24 \mathrm{sec}$ \\
\hline \hline
\end{tabular}


The sharp first-order Raman peak at $292 \mathrm{~cm}^{-1}$ corresponds to the LO phonon of $c$-GaAs, and the structures seen between 500 and $590 \mathrm{~cm}^{-1}$ correspond to secondorder (two-phonon) Raman processes in c-GaAs. Figure 1 clearly exhibits the spectral evolution with respect to the incident ion energy. The decrease of the first-order LO peak intensity, as well as of the second-order features, indicates the degradation of the near-surface crystalline quality in the bombarded samples.

A comparison of the first-order LO peak (1LO) with the second-order features shows that the second-order Raman bands are as sensitive as the 1LO line is to the crystalline quality; the ratio of the $1 \mathrm{LO}$ peak area to the area of the second-order features (the area above the smooth background which extends to high frequencies) remains the same for all of the five samples in Fig. 1. The second-order LO-phonon band ( $2 \mathrm{LO}$ band, at $580 \mathrm{~cm}^{-1}$ ) decreases in intensity with increasing damage at a rate similar to that of the $1 \mathrm{LO}$ line.

Wagner and Hoffman studied low-energy ionbombarded GaAs with resonance Raman scattering. ${ }^{5}$ Probing with a $3-\mathrm{eV}$ laser line, they found the $2 \mathrm{LO}$ band to be more sensitive to damage than the 1LO line, in contrast to the behavior observed here in Fig. 1. Strong resonance Raman effects were seen in their spectra, in which the $2 \mathrm{LO}$ band completely dominated the two-phonon region. Strong resonance Raman scattering is known to occur in GaAs when the probing photon energy is close to the $E_{1}$ electronic interband-transition energy of 2.9 eV. ${ }^{12,13}$ Although the photon energy $(2.71 \mathrm{eV})$ of the laser line used in the present investigation is close enough to $E_{1}$ to produce discernible resonance enhancement of the $1 \mathrm{LO}$ line, ${ }^{12,14}$ the $2 \mathrm{LO}$ band does not dominate the two-phonon region in Fig. 1 to nearly the extent that it dominates the spectra of Wagner and Hoffman. This likely accounts for the discrepancy between their results and those of Fig. 1 with respect to the relative sensitivity of the 2LO band. Enhanced damage sensitivity of the $2 \mathrm{LO}$ band evidently depends on being very close to resonance.

The region near the LO peak of Fig. 1 is expanded and shown in Fig. 2, again in intensity units normalized to a reference standard. A systematic intensity decrease is clearly seen with increasing $\mathrm{Ar}^{+}$-ion energy. For the sample bombarded with ions of highest ion energy, 3.89 $\mathrm{keV}$, the LO Raman intensity is only about $\frac{1}{3}$ that of c-GaAs. Also evident in Fig. 2 is that, despite the significant decrease in the LO peak intensity, the peak line shape remains essentially unchanged from that of $c$-GaAs. This is a significant difference between ionetched GaAs and ion-implanted GaAs. In the latter, a pronounced LO peak-position shift and an asymmetric line-shape broadening occur in addition to the intensity decrease. ${ }^{7,15}$ This difference is important and will be discussed further in the next section.

To reveal the damage profile in low-energy ionbombarded GaAs, we performed a series of LO-intensity measurements on ion-bombarded samples which were subsequently subjected to the wet-chemical etch described in Sec. II. The $\mathrm{Ar}^{+}$-etched GaAs samples listed in Table I were chemically etched to depths up to $600 \AA$.

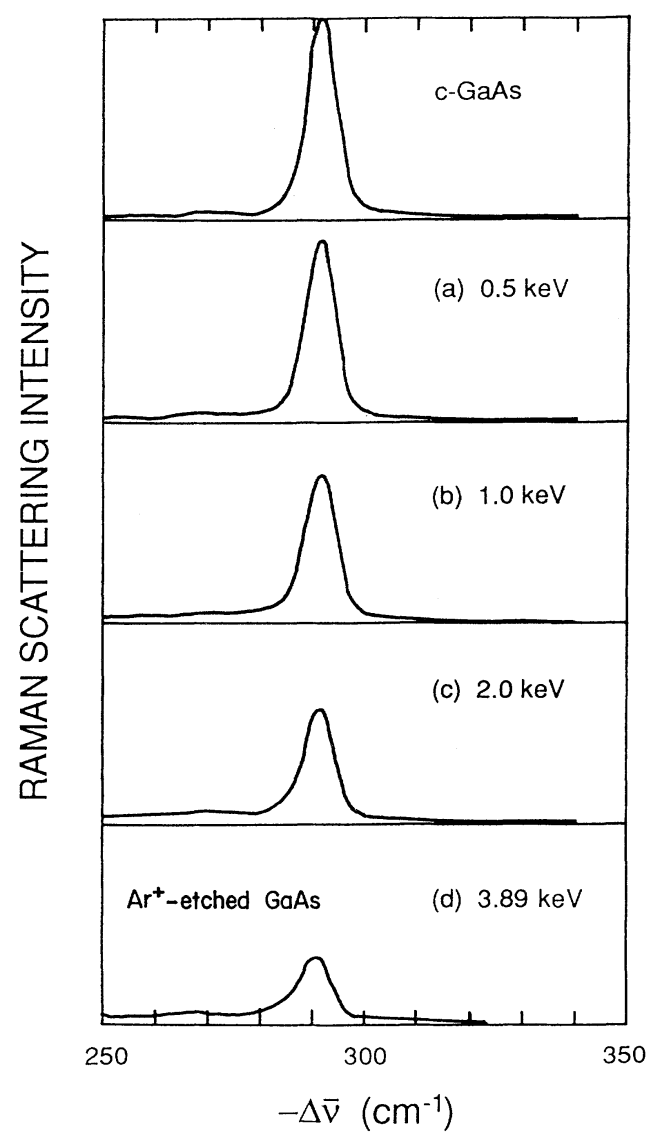

FIG. 2. Expanded view of the LO peaks of the $\mathrm{Ar}^{+}$-etched GaAs Raman spectra of Fig. 1.

By gradually removing material from the surface to a known depth, a set of samples was obtained for each bombardment energy, yielding four sets of samples. LO Raman peak-intensity measurements were then carried out on these chemically etched samples. Measurements were made with the $5145-\AA$ argon-laser line $(2.41 \mathrm{eV})$, corresponding to an optical penetration depth of $540 \AA$ for $c$-GaAs, ${ }^{10} 120 \AA$ for $a$-GaAs, ${ }^{11}$ and in between for the disordered medium. ${ }^{9}$ The LO intensity was normalized using $c$-GaAs as the reference standard. The data points (symbols) in Fig. 3 show the LO intensity versus chemical-etch depth for the four $\mathrm{Ar}^{+}$-bombarded samples. Both ion-energy dependence and depth dependence of the LO intensity are clearly demonstrated in this figure. The higher the ion energy, the lower is the LO intensity. As the surface is removed in $100-\AA$ steps, the LO intensity approaches the value characteristic of $c$-GaAs, until that value is fully recovered. For the highest bombardment energy, the undisturbed crystal is reached at an etch depth of about $600 \AA$.

The depth-dependent LO intensity exhibited in Fig. 3 reflects the depth-dependent structural change in the ion-bombarded surface layer. We will describe the bombardment-induced damage by a depth-dependent damage profile $f(z)$. The data shown in Fig. 3 can then 


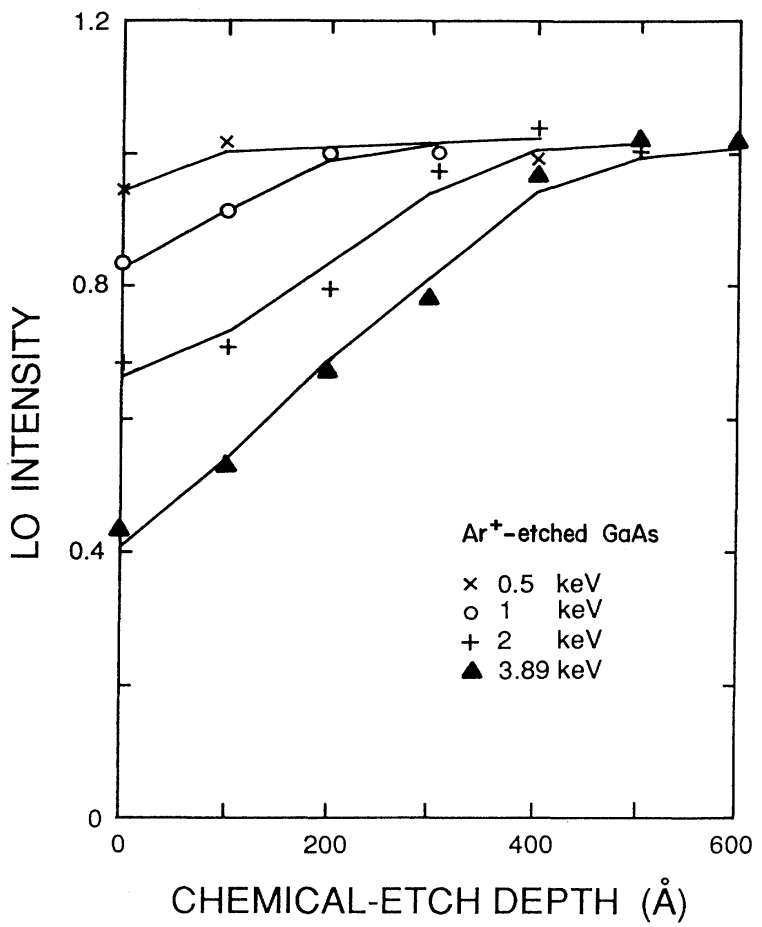

FIG. 3. The LO Raman intensity (normalized to a $c$-GaAs reference standard) of the $\mathrm{Ar}^{+}$-bombarded $\mathrm{GaAs}$ samples following chemical etching. The points (symbols) are the experimental data. The lines indicate the fits discussed in the text. For each sequence of chemically etched samples, the "fitted curve" consists of linear segments connecting points at which the fit was done (at 100- $\AA$ intervals).

be simulated using $f(z)$. In ion-bombarded solids with low ion fluence, such as $\mathrm{As}^{+}$-bombarded Si (Ref. 3) and $\mathrm{Ar}^{+}$-bombarded $\mathrm{Cu},{ }^{16}$ the damage distribution can be described by a Gaussian profile. ${ }^{17}$ For our $\mathrm{Ar}^{+}$-etched GaAs samples, the ion fluence is so high $\left(10^{17}\right.$ ions $\left./ \mathrm{cm}^{2}\right)$ that surface sputtering effects must also be taken into account. We construct $f(z)$ as follows:

$$
f(z)=\mathrm{const} \times \int_{0}^{T} \exp \left[\frac{-\left(x-r t-R_{p}\right)^{2}}{2 \sigma_{P}^{2}}\right] d t .
$$

Here $r$ is the sputtering rate, $T$ is the total sputtering time, and $d_{\text {sput }}=r T$ is the total depth of the material sputtered (or ion etched) away during the bombardment. $R_{p}$ and $\sigma_{p}$ are parameters characterizing the assumed
Gaussian and are conventionally denoted as the range and standard deviation, respectively. The depth variables $x$ and $z$ are measured from the two surface frontiers $b e$ fore and after the bombardment, and are related by

$$
x=z+d_{\text {sput }} .
$$

The sputtering rate $r$ in Eq. (1) appears only in the relation $r T=F S a^{3} / 8$, where $S$ is the sputtering yield, $F$ is the ion fluence $\left(10^{17} \mathrm{~cm}^{-2}\right.$ for these samples), and $a$ is the lattice constant of GaAs.

The function $f(z)$ contains four parameters: $d_{\text {sput }}, R_{p}$, $\sigma_{p}$, and the scaling constant. Two of the four parameters, $d_{\text {sput }}$ and $R_{p}$, can be estimated as follows. For ion energy of a few $\mathrm{keV}, S$ varies slowly with ion energy, and is about 1 atom/ion for normal incidence in $\mathrm{Ar}^{+}$. sputtered GaAs. ${ }^{18,19}$ For an angle of incidence of $45^{\circ}$, the yield is expected to be higher by a factor of about $\left(\cos 45^{\circ}\right)^{-1.5} \cdot{ }^{20} d_{\text {sput }}$ is thus estimated to be about $400 \AA$. The projected range $R_{p}$ was estimated from an extrapolation of the projected range data of Wilson and Brewer ${ }^{21}$ on argon-bombarded GaAs, yielding the values listed in the middle column of Table II.

With this model, now containing two undetermined parameters $\sigma_{p}$ and the scaling constant, the LO Raman intensity can be calculated as a function of the chemicaletch depth. The procedure assumes that the LO-line Raman-scattering intensity occurring at depth $z$ is proportional to $1-f(z)$, and it also uses $f(z)$ to estimate the optical-absorption coefficient at depth $z{ }^{7}$ The calculated intensities were then fitted to the measured data of Fig. 3. The solid curves in Fig. 3 are the fits to the data points. For example, for sample (d), the solid piecewise-linear curve shown in Fig. 3 is the two-parameter fit to the seven LO-intensity observations made after sequential chemical etching of this sample. The fitting parameters are shown in Table II. The fits are reasonable. The damage profiles $f(z)$ which correspond to the fits are the curves shown in Fig. 4. They indicate a smooth monotonic dropoff of damage versus depth.

Figure 4 also includes a linearly graded damage profile for each of the four samples. These linear profiles are fits to the Raman intensity data of Fig. 3 obtained by assuming a simple linear dropoff for $f(z)$. These fits are found to be as good as those obtained with the graded profiles derived from the sputtering-Gaussian model of Eq. (1). Thus the experiments reported here cannot distinguish between the two sets of curves in Fig. 4. (The primary experimental limitation is the difficulty in obtaining a fine

TABLE II. Damage-profile parameters used to fit the Raman intensity measurements done as a function of chemical etch (Fig. 3).

\begin{tabular}{ccccc}
\hline \hline Sample & $d_{\text {sput }}(\AA)^{\mathrm{a}}$ & $R_{p}(\AA)^{\mathrm{a}}$ & $f_{\max }$ & $\sigma_{p}(\AA)$ \\
\hline (a) & 500 & 35 & 0.04 & 30 \\
$(\mathrm{~b})$ & 500 & 40 & 0.11 & 128 \\
$(\mathrm{c})$ & 500 & 45 & 0.19 & 269 \\
(d) & 500 & 50 & 0.42 & 286 \\
\hline \hline
\end{tabular}

${ }^{\text {a }}$ Fixed. 


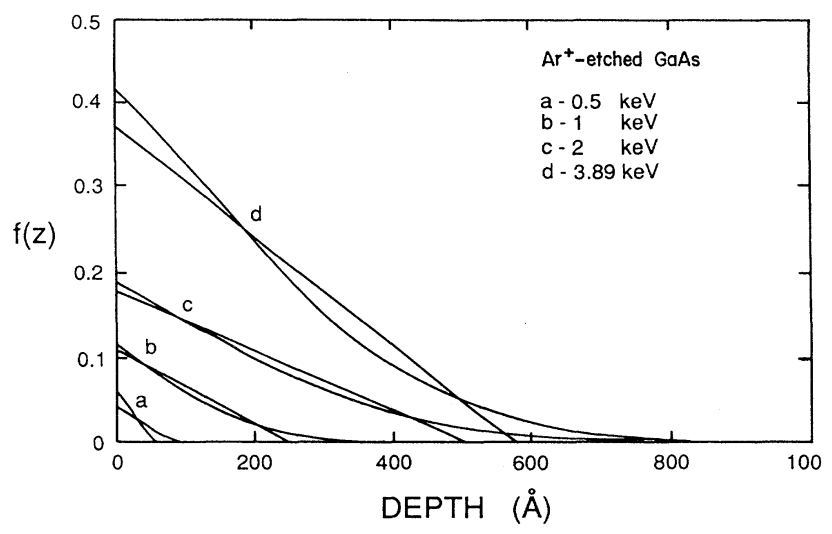

FIG. 4. Damage profile of the $\mathrm{Ar}^{+}$-bombarded GaAs samples. The curves are derived from the fits of Fig. 3 using the Gaussian-sputtering model described in the text. The straight lines are from fits to the data points of Fig. 3 using a simple linear profile with two parameters: $f_{\max }$ and $d$ (damage-layer thickness).

grid of chemical-etch depths.) Nevertheless, the smooth curves derived from the analysis based on Eq. (1) provide the more physically reasonable depth-profile results.

\section{REFLECTIVITY RESULTS AND INTERPRETATIONS OF THE DAMAGE}

It has been pointed out in the preceding section that the LO Raman peak of $\mathrm{Ar}^{+}$-etched GaAs exhibits essentially the same line shape and peak position as that of $c$-GaAs. This result is drastically different from the LO Raman peak observed in ion-implanted GaAs, where, in addition to the intensity decrease, the LO peak shifts and asymmetrically broadens. ${ }^{7,15}$ Figure 5 shows a spectroscopic comparison of the LO mode for sample (d) (3.89$\mathrm{keV} \mathrm{Ar}{ }^{+}$energy) of the ion-etched series and for a sample of ion-implanted GaAs which had been bombarded with $45-\mathrm{keV} \mathrm{Be}^{+}$ions to a fluence of $10^{14} \mathrm{~cm}^{-2}$ (see also Ref. 7). Each panel includes a comparison to the $c$-GaAs spectrum of the starting material prior to bombardment (Si-doped GaAs for $\mathrm{Ar}^{+}$-etched $\mathrm{GaAs}$ and $\mathrm{Cr}$-doped semi-insulating GaAs for $\mathrm{Be}^{+}$-etched GaAs; the two $c$-GaAs spectra are experimentally indistinguishable). The spectra were taken with the $5145-\AA(2.41-\mathrm{eV})$ argonlaser line, so that resonance effects are minimal. A1though the LO peak heights are different in the $\mathrm{Ar}^{+}$. etched $\mathrm{GaAs}$ and $\mathrm{Be}^{+}$-etched $\mathrm{GaAs}$ spectra, the integrated intensities (area under the peaks) are nearly the same, so that a detailed comparison is appropriate.

Raman-scattering studies of Holtz et al. $^{7}$ have shown that the damage layer in $\mathrm{Be}^{+}$-etched GaAs consists of a fine-scale mixture of amorphous GaAs and GaAs nanocrystals. The peak-position downshift and the asymmetric broadening, clearly seen in the lower panel of Fig. 5 , manifest finite-size effects on the vibrational excitations in the nanometer-scale (50-200 $)$ microcrystals present in $\mathrm{Be}^{+}$-etched GaAs. In contrast to this, the peak position of $\mathrm{Ar}^{+}$-etched GaAs (upper panel of Fig. 5) is scarcely shifted from the crystal peak position, and the

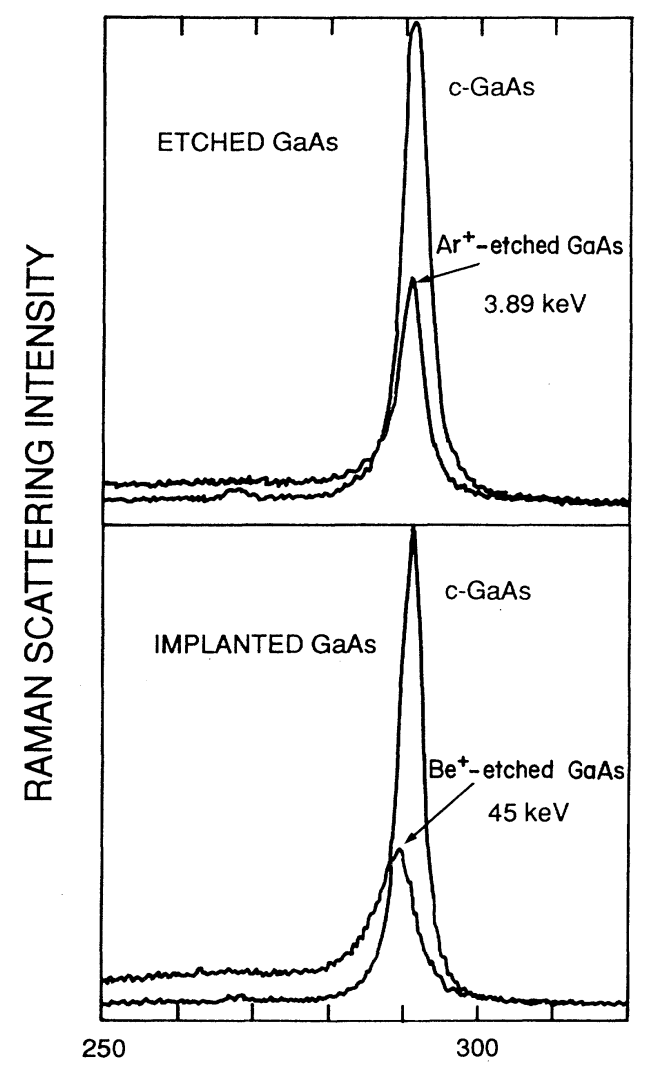

FIG. 5. Raman comparison of argon-etched GaAs and ionimplanted GaAs. The upper panel shows the LO Raman peak of sample (d) $\left(3.89-\mathrm{keV} \mathrm{Ar}{ }^{+}\right.$ions, $10^{17}$ ions $\left./ \mathrm{cm}^{2}\right)$. The lower panel shows the $\mathrm{LO}$ peak of $\mathrm{Be}^{+}$-implanted $\mathrm{GaAs}\left(45-\mathrm{keV} \mathrm{Be}^{+}\right.$ ions, $10^{14}$ ions $/ \mathrm{cm}^{2}$ ). Both panels include the $c$-GaAs spectrum of the virgin crystal prior to ion bombardment.

line shape is also very close to that of $c$-GaAs. More precisely, the peak shift in $\mathrm{Ar}^{+}$-etched GaAs is less than 0.5 $\mathrm{cm}^{-1}$, compared with $2.0 \mathrm{~cm}^{-1}$ in $\mathrm{Be}^{+}$-etched GaAs; and no peak broadening [increase in full width at half maximum (FWHM)] is seen in $\mathrm{Ar}^{+}$-etched GaAs, while it is $3.0 \mathrm{~cm}^{-1}$ in $\mathrm{Be}^{+}$-etched GaAs. The absence of both a shift and a broadening in the $\mathrm{Ar}^{+}$-etched GaAs spectrum indicates that a microcrystal model is inappropriate for the crystalline phase in the ion-etched GaAs. The fact that the only bombardment-induced effect in the spectrum of $\mathrm{Ar}^{+}$-etched GaAs is the decrease in the peak height suggests that the crystalline phase in the disordered near-surface layer preserves the vibrational properties of the bulk crystal.

Figure 6 displays the uv reflectivity spectra $R(E)$ observed for the $\mathrm{Ar}^{+}$-etched GaAs samples. Also shown, as benchmark curves repeated in each panel, are the spectra of crystalline GaAs (c-GaAs) and amorphous GaAs (a-GaAs). The $c$-GaAs spectrum is derived from the ellipsometry work of Aspnes and Studna, ${ }^{10}$ while the $a$ GaAs spectrum is derived from ellipsometry studies of implantation-amorphized GaAs. ${ }^{11}$ Indicated in each panel is the $\mathrm{Ar}^{+}$-ion kinetic energy used in the ion bom- 
bardment. The reflectivity spectra in this photon-energy region are dominated by direct (k-vector conserved) interband electronic transitions. The three well-defined peaks of the spectrum of $c$-GaAs are conventionally denoted as $E_{1}$ (at $\left.2.9 \mathrm{eV}\right), E_{1}+\Delta_{1}$ (at $3.1 \mathrm{eV}$, the spinorbit-split partner of $E_{1}$ ), and $E_{2}$ (at $5.0 \mathrm{eV}$ ). The $E_{1} /\left(E_{1}+\Delta_{1}\right)$ doublet is attributed to direct interband transitions along the $\Lambda$ directions and near the $L$ symmetry points of the Brillouin zone; the $E_{2}$ peak is associated with transitions at the plateau along the $\Sigma$ directions and near the $X$ points. $^{22}$

Lorentz-oscillator analysis ${ }^{9}$ of the reflectivity spectra in Fig. 6 was carried out to deduce the dielectric functions, and then other optical properties, of the ion-etched samples. Figures 7 and 8 show the real and imaginary parts of the dielectric functions of these samples. The oscillator parameters corresponding to the fit obtained for sample (d) are given in Table III, together with the parameters for the starting crystal. It should be emphasized that the near-surface structure of the bombarded samples is not uniform but instead is strongly depth dependent (the structure changes significantly in a distance corresponding to the optical penetration depth), as was shown in Sec. III. The dielectric functions shown in Figs. 7 and 8 should thus be understood as pseudodielectric functions. ${ }^{23}$

Appreciable effects of ion bombardment on the interband spectrum can be seen even for the sample with lowest ion energy, $0.5 \mathrm{keV}$. As the ion energy increases, the $E_{2}$ peak height decreases substantially, while the $E_{1} /\left(E_{1}+\Delta_{1}\right)$ doublet peak height changes little. All of

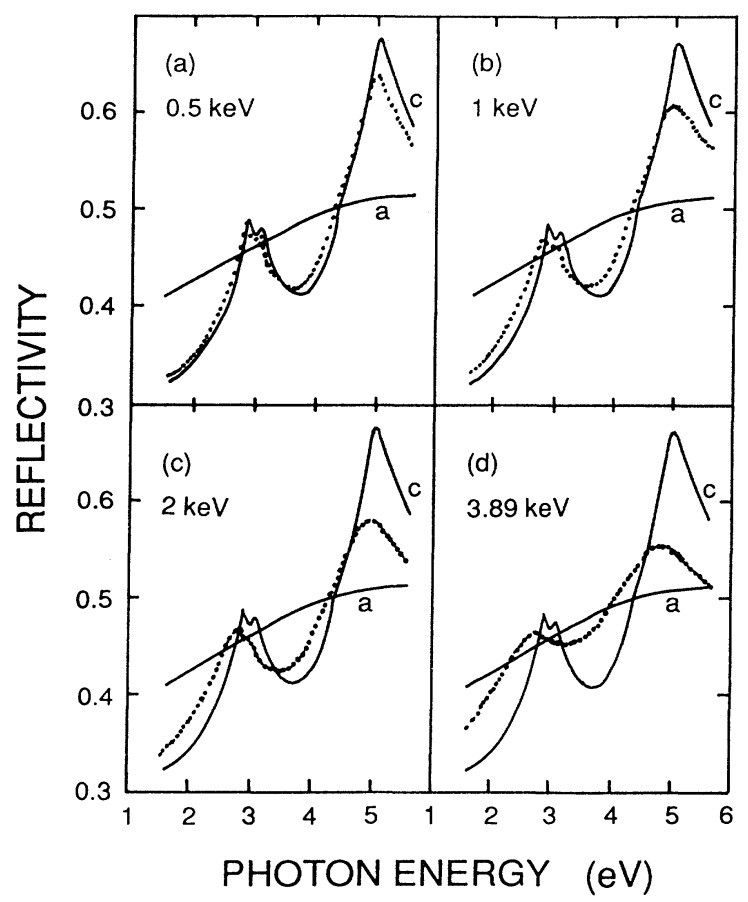

FIG. 6. Reflectivity spectra, shown as points, of the $\mathrm{Ar}^{+}$bombarded GaAs samples. Repeated in each panel are the spectra of $c$-GaAs and $a-\mathrm{GaAs}$ for comparison.

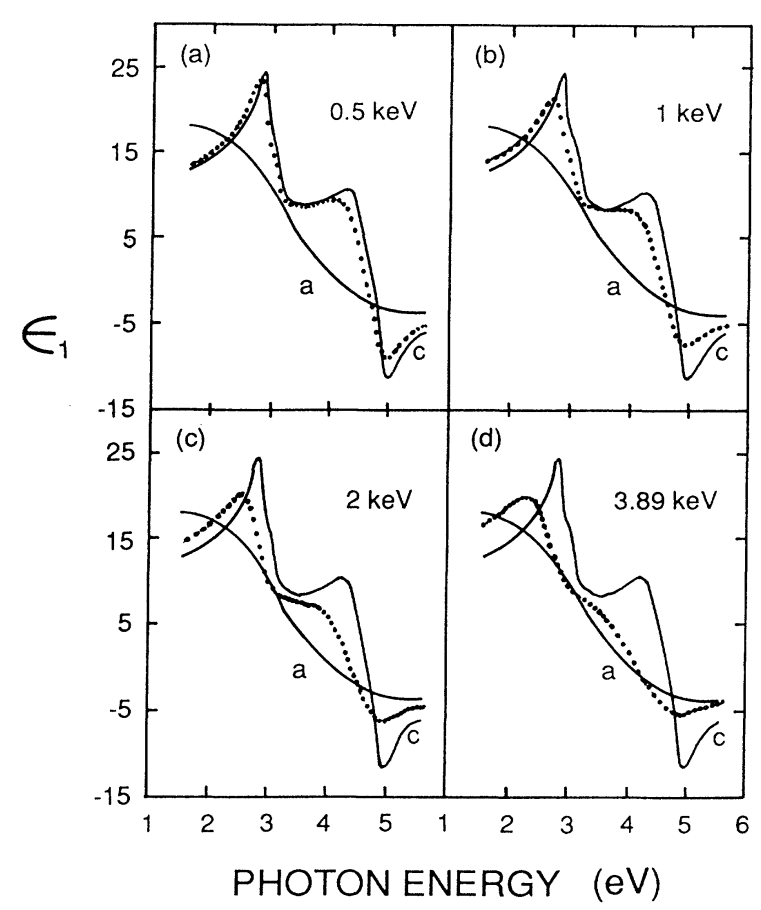

FIG. 7. Real part of the dielectric function of the $\mathrm{Ar}^{+}$bombarded GaAs samples, obtained from the reflectivity spectra of Fig. 6 using oscillator analysis. The corresponding spectra for $c$-GaAs and $a$-GaAs are included for comparison.

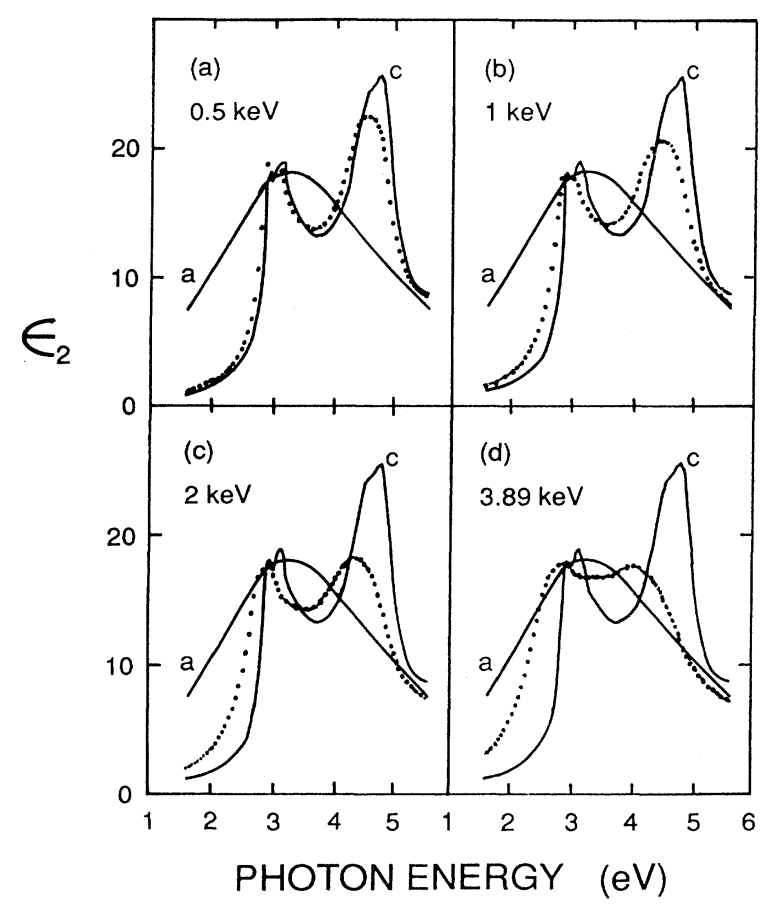

FIG. 8. Imaginary part of the dielectric function of the $\mathrm{Ar}^{+}$-bombarded GaAs samples, along with the $c$-GaAs and $a$ GaAs counterparts. 
TABLE III. Oscillator parameters fitted to the uv reflectivity of sample (d) (ion etched with 3.89-keV $\mathrm{Ar}^{+}$ions), compared to the parameters fitted to the uv reflectivity of the starting crystal. The parameter values listed in the table have been rounded off from the actual fitting parameters.

\begin{tabular}{ccccccc}
\hline $\begin{array}{c}\text { Interband } \\
\text { transition }\end{array}$ & $c$-GaAs & $\begin{array}{c}E_{i}(\mathrm{eV}) \\
\mathrm{Ar}^{+} \text {-etched GaAs }\end{array}$ & $c$-GaAs & $\begin{array}{c}\Gamma_{i}(\mathrm{eV}) \\
\mathrm{Ar}^{+} \text {-etched GaAs }\end{array}$ & $c$-GaAs & $\begin{array}{c}F_{i}(\mathrm{eV}) \\
\mathrm{Ar}^{+} \text {-etched GaAs }\end{array}$ \\
\hline$E_{1}$ & 2.92 & 2.64 & 0.19 & 0.86 & 2.23 & 4.44 \\
$E_{1}+\Delta_{1}$ & 3.12 & 2.94 & 0.34 & 0.79 & 3.30 & 3.62 \\
& 3.41 & 3.40 & 0.65 & 0.87 & 3.65 & 3.86 \\
& 3.89 & 3.90 & 1.05 & 1.05 & 4.80 & 4.53 \\
& 4.55 & 4.30 & 0.74 & 1.36 & 7.45 & 7.08 \\
$E_{2}$ & 4.84 & 4.66 & 0.36 & 0.93 & 4.40 & 2.92 \\
& 5.87 & 6.01 & 1.70 & 2.90 & 7.65 & 8.53 \\
& & & & $\epsilon_{\infty}=1.5$ & $\epsilon_{\infty}=1.5$ \\
\hline \hline
\end{tabular}

the peaks broaden, and the spectrum tends toward that of $a$-GaAs as ion energy increases.

There is an additional, and very striking, feature of Figs. 6, 7, and 8. Along with the broadenings of the $E_{1}$, $E_{1}+\Delta_{1}$, and $E_{2}$ peaks, the positions of all three peaks shift to lower photon energies. The oscillator energies corresponding to $E_{1}, E_{1}+\Delta_{1}$, and $E_{2}$ in Table III [for sample (d)] also clearly exhibit such red shifts. For the 3.89-keV argon-etched [sample (d)], the energies are red shifted by about $0.2 \mathrm{eV}$ with respect to the crystal. This behavior is very different from that seen for the interband reflectivity in $\mathrm{Be}^{+}$-implanted $\mathrm{GaAs}$ (Ref. 9) and $\mathrm{Si}^{+}$implanted GaAs. ${ }^{24}$ In ion implantation, the predominant effect is peak broadening; there is no discernible shift in peak position. ${ }^{9}$

Figure 9 compares the interband reflectivity spectra of $\mathrm{Ar}^{+}$-etched GaAs [sample (d)] and $\mathrm{Be}^{+}$-etched $\mathrm{GaAs}$ (45-keV, $10^{14}$ ions $/ \mathrm{cm}^{2}$, also see Ref. 9), again including the $c$-GaAs spectrum as a reference. For $\mathrm{Be}^{+}$-etched GaAs, the height of the $E_{1} /\left(E_{1}+\Delta_{1}\right)$ doublet decreases about as much as that of the $E_{2}$ peak. This suggests that a uniform disordered layer is probed throughout this photon-energy region, since the optical penetration depth $1 /(2 \alpha)$ is twice as large $(70 \AA)$ for the $E_{1} /\left(E_{1}+\Delta_{1}\right)$ doublet as for the $E_{2}$ peak ( $30 \AA$ ) in $\mathrm{Be}^{+}$-etched GaAs. ${ }^{9}$ This argument thus suggests that the damage layer in $\mathrm{Be}^{+}$. etched GaAs is uniform to depths in excess of $70 \AA$, which is in fact the case (uniform high damage extends to $1500 \AA$ in this material ${ }^{7}$ ). For $\mathrm{Ar}^{+}$-etched GaAs, a similar, or slightly larger, penetration-depth ratio is expected between the $E_{1} /\left(E_{1}+\Delta_{1}\right)$ and the $E_{2}$ peaks, but here we see a greater decrease in peak height for $E_{2}$ than for $E_{1} /\left(E_{1}+\Delta_{1}\right)$, indicating a higher level of damage in the first $30 \AA$ than in the first $70 \AA$. This is consistent with the graded damage profiles of Fig. 4.

The most distinctive feature of the $\mathrm{Ar}^{+}$-etched GaAs reflectivity spectrum of Fig. 9 is the clear red shift seen for both the $E_{1} /\left(E_{1}+\Delta_{1}\right)$ and the $E_{2}$ peaks. In $\mathrm{Be}^{+}$. etched GaAs, on the other hand, such red shifts are absent, and the only spectral change is the peak broadening, which has been accounted for by the finite-size-induced lifetime decrease of the electronic excitations in the nanocrystals which make up the crystalline phase within the implanted damage layer. ${ }^{9}$

Distinct nanocrystals do not manifest themselves in our experimental results for $\mathrm{Ar}^{+}$-etched GaAs. If present, such nanocrystals would reveal themselves by a shifted and broadened LO Raman line, ${ }^{7}$ but these effects are absent in Figs. 2 and 5. The crystalline component in the argon-etch-induced damage layer appears to be con-

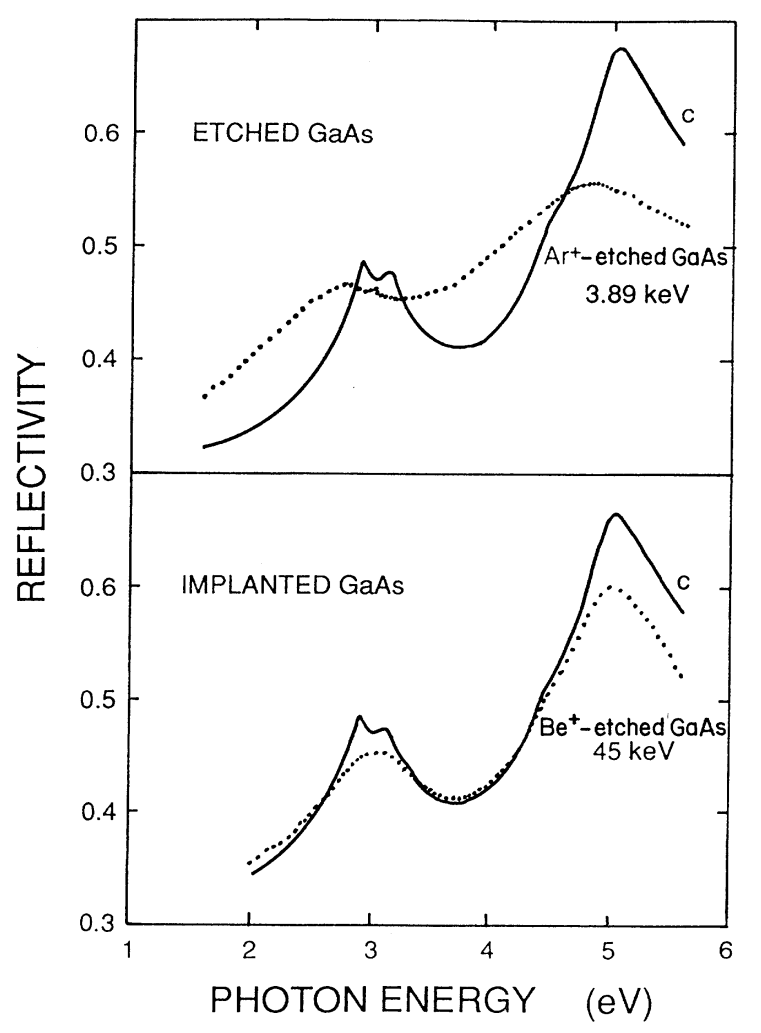

FIG. 9. Comparison of the reflectivity spectrum of argonetched GaAs and ion-implanted GaAs. Dotted curves correspond to the spectrum of the 3.89-keV $\mathrm{Ar}^{+}$-etched sample (upper panel), and the $45-\mathrm{keV} \mathrm{Be}^{+}$-implanted sample (lower panel). Both panels include the $c$-GaAs spectrum of the virgin crystal prior to ion bombardment. 
tinuous, although it is highly disordered and (from the pronounced electron-energy red shifts of Figs. 6-9) clearly different from bulk crystalline GaAs. A highly strained crystalline layer, or a crystalline layer which contains an extremely high density of point defects, are two possibilities. A high density of arsenic vacancies is a plausible candidate for the second scenario, since $\mathrm{X}$-rayphotoelectron studies of $\mathrm{Ar}^{+}$-etched GaAs show that the damage layer is arsenic deficient. ${ }^{25}$

The idea of a highly strained crystalline layer can be tested by a comparison to known effects of high pressure on interband energies and phonon frequencies in $c$-GaAs. Pressure blue shifts (shifts toward higher photon energy) the $E_{1} /\left(E_{1}+\Delta_{1}\right)$ and $E_{2}$ peaks in the tetrahedrally bonded semiconductor family to which GaAs belongs. ${ }^{26,27}$ If we assume that the red shift observed in the reflectivity spectrum of $\mathrm{Ar}^{+}$-etched GaAs (Figs. 6-8) is due to an equivalent negative pressure, then we may estimate that equivalent pressure from the observed red shifts and the known pressure coefficients. For sample (d), the most highly damaged sample, this pressure turns out to be about $-30 \mathrm{kbar}$. But this high a negative pressure would produce a very substantial $\left(-14 \mathrm{~cm}^{-1}\right)$ (Ref. 28) shift of position of the LO Raman line, and no shift is observed (Figs. 2 and 5). So the simple idea of an equivalent negative pressure does not work.

The spectral changes exhibited in Figs. 6-8, both the peak broadenings and the peak shifts, resemble effects associated with increasing temperature. Temperature effects on interband electronic properties have been investigated both experimentally and theoretically for many years. $^{29}$ Interband peaks broaden and red shift with increasing temperature. This suggests that the ion-etch damage may be viewed in terms of an equivalent temperature. The temperature dependence arises from two separate contributions, the temperature-induced volume change and the increased electron-phonon scattering. The volume change is not relevant, because its effect is the same as that of the pressure effect discussed above. The electron-defect scattering in the damaged medium may play a role similar to that of the electron-phonon scattering in the temperature effect. To estimate an equivalent temperature, we use the known temperature coefficients of the interband reflectivity peaks in $c$-GaAs near room temperature: $-0.55 \mathrm{meV} / \mathrm{K}$ for $E_{1}$ and $-0.50 \mathrm{meV} / \mathrm{K}$ for $E_{2} .{ }^{29} \mathrm{Keep}$ in mind that the volumechange contribution and the electron-phonon contribution share the temperature coefficients nearly equally, ${ }^{29}$ and that only the electron-phonon interaction contributes to the equivalent temperature. Thus the peak shifts for $E_{1}$ and $E_{2},-0.15$ and $-0.20 \mathrm{eV}$, respectively, observed for sample (d), correspond to equivalent temperatures of 550 and $800 \mathrm{~K}$. Also note that, because of the different optical penetration depth at $E_{1}$ and $E_{2}, E_{1}$-energy photons probe to a depth of about $70 \AA$ while $E_{2}$-energy photons probe to a shallower (and more disordered) depth of about $30 \AA$. Thus the difference in equivalent temperature is in the right direction (higher equivalent temperature corresponds to more disorder).

The slight shift in the LO Raman peak shown in Fig. 5 is not inconsistent with the equivalent temperature argu- ment. The temperature coefficient of the LO peak is $-1.3 \times 10^{-2} \mathrm{~cm}^{-1} / \mathrm{K}^{30} \mathrm{~A}$ down shift of $0.5 \mathrm{~cm}^{-1}$ thus corresponds to an equivalent temperature of about 380 K. The Raman spectrum in Fig. 5 was obtained with 2.41-eV laser light, which has a large penetration depth of about $300 \AA$. Thus the equivalent temperature averaged over the probed medium is expected to be substantially lower than the values obtained from the shifts of the $E_{1}$ and $E_{2}$ interband peaks. A crude estimate of about $400 \mathrm{~K}$ is obtained from the discussion of the preceding paragraph. The agreement of the equivalent temperatures estimated from the two independent experiments, though largely qualitative, supports the argument of an equivalent temperature, i.e., "frozen-in phonons" or structural defects. Taken overall, the fact that the use of an equivalent temperature mimics most of the spectral effects seen in ion-etched GaAs supports the idea that electron-defect interactions are responsible for the observed effects. As stated earlier, arsenic vacancies are likely candidates for these point defects.

\section{SUMMARY}

The optical experiments reported here have yielded information about the depth and the nature of the damage layer created in the near-surface region of GaAs by lowenergy bombardment with argon ions. The combination of sequential chemical etch and Raman scattering (Fig.3), along with the fits to these data based on the Gaussiandistribution and continuous-sputtering model [Eq. (1)], have provided the depth-profile curves of Fig. 4. The peak damage is at the surface and the graded profile shows a nearly linear damage dropoff with depth. The total damage-layer thickness varies from under $100 \AA$ for $0.5-\mathrm{keV}$ argon ions to about $600 \AA$ for $3.89-\mathrm{keV}$ ions.

The results show that the structure of the etch-induced damage layer is quite different from the structure of the deeper damage layer produced by high-energy ion implantation. For ion-implanted GaAs, the LO Raman line broadens and red shifts; for argon-etched GaAs, the LO line shape scarcely changes (Fig. 5). For ion-implanted GaAs, the electronic interband peaks broaden but do not shift; for argon-etched GaAs, pronounced red shifts are seen in addition to the broadening. The spectral changes attributed, in ion-implanted material, to finite-size effects associated with distinct nanocrystals, are absent in argon-etched GaAs, which exhibits different spectral changes. The optical effects seen in $\mathrm{Ar}^{+}$-etched GaAs bear a definite resemblance to those produced by high temperature, suggesting densely distributed disorder. The tentative picture which emerges is a continuous crystalline layer containing a very high density of point defects. Previous XPS studies suggest that arsenic vacancies provide a plausible possibility for these defects.

\section{ACKNOWLEDGMENTS}

The authors wish to thank M. Holtz, L. C. Burton, and E. Cole for valuable discussions. We owe a great debt to D. M. Hoffman for her generous help and invaluable advice with spectroscopic instrumentation. This work was supported in part by Texas Instruments and the Virginia Center for Innovative Technology. 
*Present address: Coordinated Science Laboratory, University of Illinois, Urbana, IL.

$\dagger$ Present address: Alcoa Laboratories, Alcoa Center, PA.

${ }^{1}$ S. W. Pang, Solid State Tech. 27, 249 (1984); S. W. Pang, J. Electrochem. Soc. 133, 784 (1986).

${ }^{2}$ E. Cole, Ph.D. dissertation, Virginia Tech, 1988.

${ }^{3}$ X. C. Mu, S. J. Fonash, B. Y. Yang, K. Vedam, A. Rohatgi, and J. Rieger, J. Appl. Phys. 58, 4282 (1985).

${ }^{4}$ J. B. Clegg, Surf. Interface Anal. 10, 332 (1987).

5J. Wagner, and Ch. Hoffman, Appl. Phys. Lett. 50, 682 (1987).

${ }^{6} \mathrm{~W}$. McLevige, Ph.D. dissertation, University of Illinois, Urbana, 1978.

${ }^{7}$ M. Holtz, R. Zallen, O. Brafman, and S. Matteson, Phys. Rev. B 37, 4609 (1988).

${ }^{8}$ W. Hayes and R. Loudon, Scattering of Light by Crystals (Wiley, New York, 1978).

${ }^{9}$ G. F. Feng and R. Zallen, Phys. Rev. B 40, 1064 (1989).

${ }^{10}$ D. E. Aspnes and A. A. Studna, Phys. Rev. B 27, 985 (1983).

${ }^{11}$ D. E. Aspnes (private communication); also see D. E. Aspnes, S. M. Kelso, C. G. Olson, and D. W. Lynch, Phys. Rev. Lett. 48, 1863 (1982).

${ }^{12}$ M. H. Grimsditch, D. Olego, and M. Cardona, Phys. Rev. B 20, 1758 (1979).

${ }^{13}$ R. Trommer and M. Cardona, Phys. Rev. B 17, 1865 (1978).

${ }^{14} \mathrm{M}$. Cardona, in Light Scattering in Solids II, edited by M. Cardona and G. Güntherodt, Topics in Applied Physics Vol. 50 (Springer, Berlin, 1982), p. 19.

${ }^{15}$ H. Richter, Z. P. Wang, and L. Ley, Solid State Commun. 39, 625 (1981).

${ }^{16}$ C. A. English and M. L. Jenkins, in Astrophysics, Chemistry, and Condensed Matter, edited by D. A. Bromley (Plenum, New York, 1985), Vol. 6, p. 325.
${ }^{17}$ J. Lindhard, M. E. Scharff, and H. E. Schioett, K. Dan. Vidensk. Selsk. Mat. Fys. Medd. 13 (14), 42 (1963).

${ }^{18}$ J. M. Poate, in Astrophysics, Chemistry, and Condensed Matter (Ref. 16), p. 133.

${ }^{19}$ M. Kawable, N. Masuda, and S. Namba, Appl. Opt. 17, 2556 (1978).

${ }^{20}$ R. E. Johnson, B. Sundquist, P. Hakansson, M. Salehpour, and G. Save, Surf. Sci. 179, 187 (1987).

${ }^{21}$ R. G. Wilson and G. R. Brewer, Ion Beam: with Application to Ion Implantation (Krieger, Malabar, Florida, 1979).

${ }^{22}$ M. L. Cohen and J. R. Chelikowsky, in Handbook on Semiconductors 1, edited by W. Paul (North-Holland, Amsterdam, 1982), p. 219; J. R. Chelikowsky and M. L. Cohen, Phys. Rev. B 14, 556 (1976).

${ }^{23}$ D. E. Aspnes, Thin Solid Films 89, 249 (1982).

${ }^{24}$ G. F. Feng, Ph.D. dissertation, Virginia Tech, 1989.

${ }^{25}$ J. M. Epp, J. G. Dillard, A. Siochi, R. Zallen, S. Sen, and L. C. Burton, Chem. Mater. 2, 173 (1990).

${ }^{26}$ R. Zallen and W. Paul, Phys. Rev. 155, 703 (1967).

${ }^{27} \mathrm{G}$. Martinez, in Handbook on Semiconductors 2, edited by $\mathbf{M}$. Balkanski (North-Holland, Amsterdam, 1980), p. 181.

${ }^{28}$ B. A. Weinstein and R. Zallen, in Light Scattering in Solids V, edited by M. Cardona and G. Güntherodt, Topics in Applied Physics Vol. 54 (Springer-Verlag, Berlin, 1982), p. 463.

${ }^{29}$ M. L. Cohen and D. J. Chadi, in Handbook on Semiconductors 2, edited by M. Balkanski (North-Holland, Amsterdam, 1980), p. 155; P. Lautenschlager, P. B. Allen, and M. Cardona, Phys. Rev. B 31, 2163 (1985); P. Lautenschlager, M. Garriga, S. Logothetidis, and M. Cardona, ibid. 35, 9174 (1987).

${ }^{30} \mathrm{~J}$. Sapriel, J. Chavignon, F. Alexandre, and R. Azoulay, Phys. Rev. B 34, 7118 (1986). 